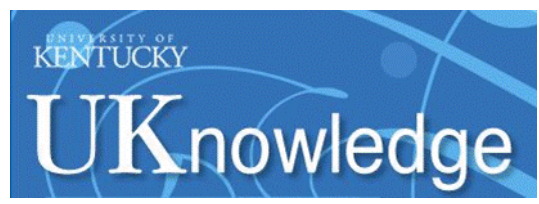

University of Kentucky

UKnowledge

\title{
The AGN-obscuring Torus: The End of the "Doughnut" Paradigm?
}

\author{
Moshe Elitzur \\ University of Kentucky, moshe@pa.uky.edu \\ Isaac Shlosman \\ University of Kentucky
}

Follow this and additional works at: https://uknowledge.uky.edu/physastron_facpub

Part of the Astrophysics and Astronomy Commons, and the Physics Commons

Right click to open a feedback form in a new tab to let us know how this document benefits you.

\section{Repository Citation}

Elitzur, Moshe and Shlosman, Isaac, "The AGN-obscuring Torus: The End of the "Doughnut" Paradigm?" (2007). Physics and Astronomy Faculty Publications. 210.

https://uknowledge.uky.edu/physastron_facpub/210

This Article is brought to you for free and open access by the Physics and Astronomy at UKnowledge. It has been accepted for inclusion in Physics and Astronomy Faculty Publications by an authorized administrator of UKnowledge. For more information, please contact UKnowledge@lsv.uky.edu. 


\section{The AGN-obscuring Torus: The End of the "Doughnut" Paradigm?}

Digital Object Identifier (DOI)

http://dx.doi.org/10.1086/508158

\section{Notes/Citation Information}

Published in The Astrophysical Journal Letters, v. 648, no. 2, p. L101-L104.

(c) 2007. The American Astronomical Society. All rights reserved.

The copyright holder has granted permission for posting the article here. 


\title{
THE AGN-OBSCURING TORUS: THE END OF THE “DOUGHNUT” PARADIGM?
}

\author{
Moshe Elitzur ${ }^{1}$ AND IsaAC Shlosman \\ Department of Physics and Astronomy, University of Kentucky, Lexington, KY 40506-0055; moshe@pa.uky.edu, shlosman@pa.uky.edu \\ Received 2006 April 17; accepted 2006 August 4; published 2006 September 5
}

\begin{abstract}
Unified schemes of active galactic nuclei (AGNs) require an obscuring dusty torus around the central engine. The compact sizes (only a few parsecs) determined in recent high-resolution observations require that the obscuring matter be clumpy and located inside the region where the black hole gravity dominates over the galactic bulge. This location is in line with the scenario depicting the torus as the region of the clumpy wind coming off the accretion disk in which the clouds are dusty and optically thick. We study here the outflow scenario within the framework of hydromagnetic disk winds, incorporating the cloud properties determined from detailed modeling of the IR emission from clumpy tori. We find that torus clouds were likely detected in recent water maser observations of NGC 3079. In the wind scenario, the AGN main dynamic channel for release of accreted mass seems to be switching at low luminosities from torus outflow to radio jets. The torus disappears when the bolometric luminosity decreases below $\sim 10^{42} \mathrm{ergs} \mathrm{s}^{-1}$ because the accretion onto the central black hole can no longer sustain the required cloud outflow rate. This disappearance seems to have been observed in both LINERs and radio galaxies. With further luminosity decrease, suppression of cloud outflow spreads radially inward from the disk's dusty, molecular region into its atomic, ionized zone, resulting in the disappearance of the broad emission-line region at lower luminosities yet to be determined.
\end{abstract}

Subject headings: galaxies: active — galaxies: nuclei — galaxies: Seyfert — infrared: galaxies — quasars: general

\section{INTRODUCTION}

The great diversity of active galactic nucleus (AGN) classes has been explained by a single unified scheme (e.g., Antonucci 1993; Urry \& Padovani 1995). The nuclear activity is powered by a supermassive $\left(\sim 10^{6}-10^{10} M_{\odot}\right)$ black hole $(\mathrm{SBH})$ and its accretion disk. This central engine is surrounded by dusty clouds, which are individually optically thick, in a toroidal structure (Krolik \& Begelman 1988). The clumpy torus is characterized by two fundamental properties:

1. Anisotropic obscuration of the central region so that sources viewed face-on are recognized as type 1 objects, those observed edge-on are type 2 . From the statistics of Seyfert 1 and 2 galaxies, the torus height ${ }^{2}$ and radius obey $H / R \sim 1$ (Schmitt et al. 2001).

2. Dust reemission in the IR of the AGN obscured radiation. This emission is the only means to find the torus radial extent since obscuration cannot separately determine $H$ and $R$.

Two approaches have been taken for the torus dynamic origin. A hydrostatic scenario depicts the torus as a doughnut-like structure populated by molecular clouds accreted from the galaxy (Krolik \& Begelman 1988). However, the origin of vertical motions capable of sustaining the clouds in a hydrostatic structure with $H \sim R$ was recognized from the start as problematic and has eluded solution thus far (e.g., Davies et al. 2006). The other scenario, based on the seminal work by Blandford \& Payne (1982), involves the outflow of clouds embedded in a hydromagnetic disk wind (Emmering et al. 1992, hereafter EBS92; Königl \& Kartje 1994; Kartje \& Königl 1996; Bottorff et al. 1997, 2000; Kartje at al. 1999; Everett 2005). In this approach the torus is merely a region in the wind that happens to provide the required toroidal obscuration; i.e., it is that region wherein the clouds are dusty and optically thick.

\footnotetext{
${ }^{1}$ On sabbatical leave at Laboratoire d'Astrophysique de Grenoble.

${ }^{2}$ The highest point that gives $\tau_{V} \sim 1$ along the line of sight.
}

Recent high-resolution IR observations indicate that the torus size might be no more than a few parsecs (Elitzur 2005 and references therein); in particular, VLTI observations of NGC 1068 show that the FWHM size of the $12 \mu \mathrm{m}$ emission is only $\sim 4$ pc (Jaffe et al. 2004). The compact sizes place the torus inside the region where the SBH gravity dominates over the galactic bulge. Consider a bulge that induces a linearly rising rotation curve with $\Omega \sim 1 \mathrm{~km} \mathrm{~s}^{-1} \mathrm{pc}^{-1}$, as is typical of AGN host galaxies (Sofue et al. 1999), and an SBH with a mass of $M_{\bullet, 7} \times 10^{7} M_{\odot}$ at its center. The SBH will dominate the gravitational motions within a radius $35\left(M_{\bullet}, 7 / \Omega_{1}^{2}\right)^{1 / 3} \mathrm{pc}$, where $\Omega_{1}=\Omega /\left(1 \mathrm{~km} \mathrm{~s}^{-1} \mathrm{pc}^{-1}\right)$. Since the torus is well within this region, its dynamic origin is determined in all likelihood by the central engine and its accretion disk, giving strong impetus to the outflow paradigm. The central accretion disk appears to be fed by a midplane influx of cold, clumpy material from the main body of the galaxy (Shlosman et al. 1990 and references therein). Approaching the center, conditions for developing hydromagnetically or radiatively driven winds above this equatorial inflow become more favorable. The disk-wind geometry provides a natural channel for angular momentum outflow from the disk and is found on many spatial scales, from protostars to AGNs (Blandford \& Payne 1982; EBS92; Ferreira 2006). Although theoretical questions involving cloud-uplift and wind-driving mechanisms remain (e.g., EBS92; Ferreira 2006), observations give ample evidence for winds and cloud motions (e.g., Arav et al. 1997; Elvis 2004) and support for a disk-wind geometry (e.g., Hall et al. 2003) in AGNs.

Clumpiness provides a natural explanation for the compactness of the IR emission from AGN tori, and detailed modeling of this emission sets phenomenological constraints on the torus cloud properties (Nenkova et al. 2002; M. Nenkova et al. 2006, in preparation; Elitzur et al. 2004; Elitzur 2005). Here we explore whether the torus outflow scenario can properly accommodate these constraints, and the corollaries for the AGN paradigm. 


\section{CLOUD PROPERTIES}

The only property of individual clouds constrained from the IR modeling is their optical depth. It should lie in the range 20 150 at the $V$ band, i.e., column densities $N_{\mathrm{H}} \sim 10^{22}-10^{23} \mathrm{~cm}^{-2}$ assuming standard dust-to-gas ratio. Clouds uplifted into the wind expand while moving away from the disk. A cloud starting with $N_{\mathrm{H}} \sim 10^{23} \mathrm{~cm}^{-2}$ ceases to partake in obscuration when its column is reduced by $\sim 100$. A cloud starting with a smaller column will rise to a smaller height before leaving the obscuring torus. Consider the tidal torque of the central SBH at a distance $r_{\mathrm{pc}}$ in parsecs, where the Keplerian period is $t_{\mathrm{K}}=3 \times 10^{4} M_{\bullet, 7}^{-1 / 2} r_{\mathrm{pc}}^{3 / 2} \mathrm{yr}$. To prevent a cloud with density $\rho=m_{p} n$ from shearing, it must be at least partially confined by its own gravity and/or the ambient magnetic field $B$. In the former case, the characteristic Jeans timescale is $t_{\mathrm{J}} \sim(G \rho)^{-1 / 2}=3 \times 10^{4} n_{7}^{-1 / 2} \mathrm{yr}$, where $n_{7}=n /\left(10^{7}\right.$ $\left.\mathrm{cm}^{-3}\right)$. Therefore, resistance to tidal shearing $\left(t_{\mathrm{J}}<t_{\mathrm{K}}\right)$ requires $n_{7}>M_{\bullet}, 7 / r_{\mathrm{pc}}^{3}$, which, for a cloud with column $N_{\mathrm{H}}=N_{\mathrm{H}, 23} \times$ $10^{23} \mathrm{~cm}^{-2}$, implies a size $a \lesssim 10^{16} N_{\mathrm{H}, 23} r_{\mathrm{pc}}^{3} / M_{\bullet, 7} \mathrm{~cm}$. The corresponding cloud mass is $M_{\mathrm{cl}} \lesssim 7 \times 10^{-3} N_{\mathrm{H}, 23} a_{16}^{2} M_{\odot}$, where $a_{16}=a /\left(10^{16} \mathrm{~cm}\right)$. The self-gravity of such a low mass cannot hold it together against dispersal by any reasonable internal velocities. However, an external magnetic field $B \sim 1.5 \sigma_{5} n_{7}^{1 / 2} \mathrm{mG}$ would suffice if the internal velocity dispersion is $1 \sigma_{5} \mathrm{~km} \mathrm{~s}^{-1}$.

Clouds with these very same properties may have been detected by Kondratko et al. (2005) in recent $\mathrm{H}_{2} \mathrm{O}$ maser observations of NGC 3079. Individual maser features detected in this source have sizes $\lesssim 2 \times 10^{16} \mathrm{~cm}$ and span the radial range $\sim 0.4-1.3 \mathrm{pc}$. Similar to other objects, most maser features in this source reside in an edge-on rotating disk. However, for the first time, four features were found significantly out of the disk plane, yet their line-ofsight velocities reflect the velocity of the most proximate side of the disk. Kondratko et al. note that this can be explained if, as proposed by Kartje et al. (1999), maser clouds rise to high latitudes above the rotating structure while still maintaining, to some degree, the rotational velocity imprinted by the parent disk. Because the detected maser emission involves cloud-cloud amplification that requires precise alignment in both position and velocity along the line of sight, the discovery of four high-latitude maser features implies the existence of many more such clouds partaking in the outflow. Densities of $\mathrm{H}_{2} \mathrm{O}$ masers are frequently quoted as $\sim 10^{8}-10^{9} \mathrm{~cm}^{-3}$, but these are the optimal densities to produce the highest possible brightness from a single cloud. Because of the scaling properties of $\mathrm{H}_{2} \mathrm{O}$ pumping (Elitzur et al. 1989), maser clouds with $n \sim 10^{7} \mathrm{~cm}^{-3}$ and $N_{\mathrm{H}} \sim 10^{23} \mathrm{~cm}^{-2}$ produce nearoptimal inversion and detected radiation in cloud-cloud amplification.

To produce a torus with the required thickness, the column density of a cloud must remain above $\sim 10^{21} \mathrm{~cm}^{-2}$ during the rise to height $H \sim r$. If the cloud expands at the speed of sound $c_{s}$ while rising with velocity $v$, then its size will increase by a factor $x \simeq 1+(r / a)\left(c_{s} / v\right)$. We expect $c_{s} / v \simeq 10^{-2}$ since $c_{s}$ is $1 \mathrm{~km} \mathrm{~s}^{-1}$ at a temperature of $100 \mathrm{~K}$ and the velocity scale of the cloud outflow is expected to be comparable to the Keplerian velocity, 208( $\left.M_{\bullet, 7} / r_{\mathrm{pc}}\right)^{1 / 2} \mathrm{~km} \mathrm{~s}^{-1}$. With $a / r \sim 10^{-3}$, the threedimensional (3D) expansion of such a cloud will cause its size to increase by a factor $x \sim 10$ and the column density $\left(\propto M_{\mathrm{cl}} / a^{2}\right)$ to decrease within the torus region by a factor $x^{2} \sim 100$, as required. Furthermore, in a cloud-loaded hydromagnetic disk wind the ambient magnetic field suppresses cloud expansion across the field lines while guiding the cloud motion. Magnetic confinement can be efficient in either 3D (e.g., the "melon seeds" model of diamagnetic clouds; Rees 1987), or 2D (e.g., the slender tubes model of paramagnetic clouds;
EBS92). In the simplest picture of constant area flux tubes and thermal expansion only along the field lines, the cloud column density remains unchanged in the longitudinal direction while decreasing as $1 / x$ in the orthogonal direction. In reality, the decrease of column density is likely to fall somewhere between the $1 / x^{2}$ of $3 \mathrm{D}$ thermal expansion and $1 / x$ of constant field. Whichever the case, magnetic effects always supplement the thermal confinement, enabling smaller and denser torus clouds.

\section{MASS OUTFLOW}

At a bolometric luminosity $L=L_{45} \times 10^{45} \mathrm{ergs} \mathrm{s}^{-1}$, the inner radius of the torus cloud distribution is $R_{i} \simeq 0.4 L_{45}^{1 / 2} T_{1500}^{-2.6} \mathrm{pc}$, where $T_{1500}$ is the dust sublimation temperature normalized to $1500 \mathrm{~K}$. This distance was determined from the dust temperature on the illuminated face of an optically thick cloud of composite dust representing a standard mix of Galactic grains (M. Nenkova et al. 2006, in preparation). Some dust components can exist closer than $R_{i}$, and attenuation of the central UV radiation could also reduce $R_{i}$ at low latitudes. The torus outer radius is $Y R_{i}$, with $Y \sim 5-10$. The quantity constrained by IR modeling is the number of clouds per unit length, $N_{\mathrm{cl}}=n_{\mathrm{cl}} A_{\mathrm{cl}}$, where $n_{\mathrm{cl}}$ is the number of clouds per unit volume and $A_{\mathrm{cl}}$ the cloud cross-sectional area. Neither $n_{\mathrm{cl}}$ nor $A_{\mathrm{cl}}$ enter independently into the calculation of the torus emission. An analytic expression for $N_{\mathrm{cl}}(r, \beta)$ in terms of radial distance $r$ and angle $\beta$ from the equatorial plane that agrees reasonably with all observations, and can serve as a "standard" cloud distribution, is $N_{\mathrm{cl}} \propto \mathcal{N}_{0}$ $r^{-2} \exp \left(-\beta^{2} / \sigma^{2}\right)$. Here $\mathcal{N}_{0}(\sim 5-10)$ is the total number of clouds encountered, on average, along a radial equatorial ray, and $\sigma$ $\left(\sim 45^{\circ}\right)$ measures the angular width of the cloud distribution. This phenomenological parameterization of the torus cloud distribution was not meant to account for the underlying disk, where the clouds originate, and should hold only at inclinations $\beta$ above the disk surface. In practice, we neglect the disk thickness and utilize this expression at all $\beta$.

The mass density of clouds is $M_{\mathrm{cl}} n_{\mathrm{cl}}=m_{p} N_{\mathrm{H}} N_{\mathrm{cl}}$, and the cloud outflow rate generating the torus is $m_{p} N_{\mathrm{H}}^{p} \int N_{\mathrm{cl}} v_{\mathrm{cl}} d A$. The integration is over the disk area where the torus clouds are injected out of the plane with velocity $v_{\mathrm{cl}}$. This velocity can be estimated from the dispersion velocity of molecular material in the disk - an outflow is established when the velocity of the ordered motion becomes comparable to that of the local random motions. From the maser observations in NGC 3079 Kondratko et al. (2005) find that the velocity dispersion in a small region $\left(\leq 5 \times 10^{16} \mathrm{~cm}\right)$ of strong emission is only $\sim 14 \mathrm{~km} \mathrm{~s}^{-1}$, which can be considered typical of the local random motions. Therefore, we parameterize the injection velocity as $v_{\mathrm{cl}}(r)=$ $10 v_{6} u(r) \mathrm{km} \mathrm{s}^{-1}$, where $u=v(r) / v\left(R_{i}\right)$ and $v_{6}$ is the velocity at the inner radius $R_{i}$ normalized to $10 \mathrm{~km} \mathrm{~s}^{-1}$; both $v_{6}$ and $u(r)$ are expected to be of order unity. The torus mass outflow rate in the form of clouds is thus

$$
\dot{M}_{w, \mathrm{cl}}=0.02 L_{45}^{1 / 2} T_{1500}^{-2.6} N_{\mathrm{H}, 23}^{\mathrm{tot}} v_{6} I_{1} M_{\odot} \mathrm{yr}^{-1} .
$$

Here $N_{\mathrm{H}, 23}^{\text {tot }}=\mathcal{N}_{0} N_{\mathrm{H}, 23}$ is the column density through all clouds in the equatorial plane in units of $10^{23} \mathrm{~cm}^{-2}$ and $I_{1}=$ $\int_{1}^{Y}(u / y) d y Y /(Y-1)$ is an unknown factor of order unity. The mass inflow rate across the torus is related to the bolometric luminosity via $\dot{M}_{\text {acc }}=0.02 L_{45} / \epsilon M_{\odot} \mathrm{yr}^{-1}$, where $\epsilon$ is the accretion efficiency at the torus radii. Note that $\dot{M}_{\text {acc }}$ is a function of $r$, since the mass loss in a hydromagnetic wind can be a significant fraction of the disk accretion rate; at radii characteristic of the torus, $\dot{M}_{\text {acc }}$ can be substantially larger, and $\epsilon$ 
substantially smaller, than in the innermost accretion disk (EBS92). The results for $\dot{M}_{w, \text { cl }}$ and $\dot{M}_{\text {acc }}$ yield

$$
\frac{\dot{M}_{w, \mathrm{cl}}}{\dot{M}_{\mathrm{acc}}}=\epsilon N_{\mathrm{H}, 23}^{\mathrm{tot}} v_{6} L_{45}^{-1 / 2} T_{1500}^{-2.6} I_{1} .
$$

When $L$ decreases, $\dot{M}_{w, \text { cl }} / \dot{M}_{\text {acc }}$ increases. However, this ratio cannot exceed unity. Moreover, the disk wind applies a torque on the underlying accretion disk because each magnetic field line can be considered rigid from its footpoint $r_{0}$ and up to the Alfvén radius, $r_{\mathrm{A}}$. For a total outflow rate $\dot{M}_{w}$ of a continuous wind loaded with clouds, angular momentum conservation gives $\gamma \equiv \dot{M}_{w} / \dot{M}_{\text {acc }} \sim$ $\left(r_{0} / r_{\mathrm{A}}\right)^{2}$, with $\gamma \sim 0.1-0.25$ either constant (EBS92) or weakly dependent on $r_{0}$ (Pelletier \& Pudritz 1992). Therefore, $\dot{M}_{w, \mathrm{cl}} \lesssim \gamma \dot{M}_{\text {acc }}$. When the luminosity is decreasing, eventually this bound is violated by equation (2) and the system can no longer sustain the cloud outflow rate required by the torus wind. That is, the torus should disappear in low-luminosity AGNs. If we take $\gamma \simeq 0.2$, an accretion efficiency $\epsilon \simeq 0.01$ (EBS92) and all other parameters $\sim 1$, the torus disappearance should occur at bolometric luminosities below $\sim 10^{42} \mathrm{ergs} \mathrm{s}^{-1}$. The timescale for this is short. The overall mass in torus clouds is $M_{\mathrm{tot}}=m_{p} N_{\mathrm{H}} \int N_{\mathrm{cl}} d V \simeq$ $10^{3} N_{\mathrm{H}, 23}^{\text {tot }} L_{45} Y . M_{\odot}$, so the depletion timescale when $M_{w, \mathrm{cl}} \simeq$ $\gamma \dot{M}_{\text {acc }}$ is $M_{\text {tot }} / M_{w, \text { cl }} \simeq 5 \times 10^{4}(\epsilon / \gamma) Y N_{\mathrm{H}, 23}^{\text {tot }}$ yr. Since $\epsilon Y / \gamma \sim 1$, the torus will disappear within a few Keplerian orbits.

The kinetic luminosity $E_{k}$ of the torus cloud outflow is generally insignificant. A calculation similar to that for $\dot{M}_{w, \text { cl }}$ yields $\dot{E}_{k}=$ $7 \times 10^{35} L_{45}^{1 / 2} N_{\mathrm{H}, 23}^{\text {tot }} v_{6}^{3} I_{3}$ ergs $\mathrm{s}^{-1}$, where $I_{3}=\int_{1}^{w}\left(u^{3} / y\right) d y Y /(Y-$ $1)$ is of order unity. This is negligible in the overall energy budget of the AGN. Finally, the volume-averaged density of the clouds is $\phi n$, where $\phi=a N_{\mathrm{cl}}$ is their volume filling factor. The initial outflow velocity of clouds is roughly 10 times larger than their internal velocity dispersion. Since we have $\phi \sim 10^{-2}$, the average kinetic energy is similar for the cloud outflow and the internal motions. Therefore, the assumption that the field limiting the clouds expansion is also guiding their motion is self-consistent.

\section{SUMMARY AND DISCUSSION}

The analysis presented here shows that the disk wind scenario is compatible with cloud properties inferred from models of the torus IR emission. Furthermore, the very same dusty, molecular clouds uplifted from the disk appear to have been detected in water maser observations of NGC 3079 (Kondratko et al. 2005). Proper-motion measurements, if possible, of these high-latitude masers would be extremely valuable. Detection of maser polarization seems less likely because it would require proper alignment of the magnetic fields among maser clouds that amplify each other (Elitzur 1996). Colocation of water masers and the torus also seems to be observed in NGC 1068, where, as noted by Poncelet et al. (2006), the two are at similar radial distances. The masers there trace a thin, edge-on rotating disk between 0.65 and 1.1 pc (Greenhill \& Gwinn 1977; Gallimore et al. 2001). It is intriguing that molecular structures somewhat resembling the maser disk, and which might be its extension, are also seen on much larger scales in NGC 1068. Galliano et al. (2003) find that $\mathrm{H}_{2}$ and $\mathrm{CO}$ emissions come from clouds in a disk close to edge-on with radius $140 \mathrm{pc}$ and scale height $20 \mathrm{pc}$, for $H / R \sim 0.15$. From CO mapping, Schinnerer et al. (2000) conclude that $H \sim 9-10 \mathrm{pc}$ at radial distance of $70 \mathrm{pc}$, for a similar $H / R$. Detailed, high-resolution mapping of the entire region between $\sim 1$ and $50 \mathrm{pc}$ around the nucleus can shed light on the variation in the disk structure during the transition from gravitational domination by the galactic bulge to the black hole, and on the origin of the disk wind.

Detectable only in edge-on AGNs, the water masers reside in the inner molecular regions of the accretion disk. At smaller radii, the disk composition switches from dusty and molecular to atomic and ionized (e.g., EBS92; Kartje et al. 1999). From time variations of $\mathrm{X}$-ray obscuration that indicate cloud motions across the line of sight, Risaliti et al. $(2002,2005)$ find that some of the obscuring clouds are at radii considered typical of the AGN broad-line region (BLR). Clouds at the very inner edge of the molecular disk are protected there from the central UV. Upon injection into the wind and exposure to the AGN radiation, these clouds can be responsible for the broad emission lines at low altitudes above the disk, and for the warm absorption at higher altitudes. Indeed, the kinematics of such clouds has been shown to fit the BLR line profiles (EBS92) nicely. Clouds uplifted farther out will have their entire trajectories at $r>R_{i}$ and form the obscuring torus. Since cloud properties must span some range, the torus structure is likely stratified. Clouds that reach the maximal height $H \sim r$ must start with a size of $\sim 10^{16} \mathrm{~cm}$ and column density of $\sim 10^{23} \mathrm{~cm}^{-2}$. Clouds with smaller initial size or column will lose the required obscuration at lower heights.

The fraction of type 2 objects appears to decrease with $L$, in accordance with the "receding torus" model (Simpson 2005). A decrease of $H / R$ with $L$ arises naturally in the torus outflow scenario. The torus radial dimensions are set from its inner radius $R_{i}$ and increase with $L$ due to dust sublimation. On the other hand, since outflow velocities are expected to decrease with $r$, thermal expansion will reduce the cloud column density below the range of torus clouds at $H<r$ if the relative size $a / r$ is roughly constant. An additional possible factor limiting the height at larger $L$ is bending of the streamlines by radiation pressure (e.g., de Kool \& Begelman 1995).

A key prediction of the wind scenario is that the torus disappears at low bolometric luminosities $\left(\lessgtr 10^{42} \mathrm{ergs} \mathrm{s}^{-1}\right)$ because mass accretion can no longer sustain the required cloud outflow rate, i.e., the large column densities. Observations seem to corroborate this prediction. In a Hubble Space Telescope study of a complete sample of low-luminosity $\left(\$ 10^{42} \mathrm{ergs} \mathrm{s}^{-1}\right)$ FR I radio galaxies, Chiaberge et al. (1999) detected the compact core in $85 \%$ of sources and argued that this high detection rate implies the absence of an obscuring torus. This finding was enhanced when Whysong \& Antonucci (2004 and references therein) demonstrated that M87, one of the sample sources, indeed does not contain an obscuring torus by placing stringent limits on its thermal IR emission; a similar conclusion was reached by Perlman et al. (2001). Additional evidence comes from UV monitoring of LINERs with $L \lesssim 10^{42} \mathrm{ergs} \mathrm{s}^{-1}$ by Maoz et al. (2005). By detecting variability in most of the monitored objects they demonstrated that the AGN makes a significant contribution to the UV radiation in each source and that it is relatively unobscured in both type 1 and type 2 LINERs. The histogram of UV colors shows an overlap between the two populations, with the difference between the peaks corresponding to dust obscuration in the type 2 LINERs of only $\sim 1$ mag in the $R$ band. Such toroidal obscuration is minute in comparison with higher luminosity AGNs.

The predicted torus disappearance at low $L$ does not imply that the cloud component of the disk wind is abruptly extinguished, only that its outflow rate is less than required by the "standard" IR emission observed in quasars and high-luminosity Seyfert galaxies. When $\dot{M}_{w, \text { cl }}$ drops below standard torus values, which at each AGN might occur at somewhat different $L$ because of the intrinsic spread of parameters in equation (2) $(\epsilon, v$, etc.), the outflow 
still provides toroidal obscuration as long as its column exceeds $\sim 10^{21} \mathrm{~cm}^{-2}$. Indeed, Maoz et al. find that some liners do have obscuration but much smaller than standard. Line transmission through a low-obscuration torus might also explain the low polarizations of broad $\mathrm{H} \alpha$ lines observed by Barth et al. (1999) in some low-luminosity systems. The properties of thermal IR emission from such low-luminosity AGNs should differ from the standard, a prediction that should be tested in observations.

If the toroidal obscuration were the only component removed from the system, all low-luminosity AGNs would become type 1 sources. In fact, among the LINERs monitored and found to be variable by Maoz et al. (2005), there were both sources with broad $\mathrm{H} \alpha$ wings (type 1 ) and those without (type 2). Since all objects are relatively unobscured, the broad-line component is truly missing in the type 2 sources in this sample. Similarly, Laor (2003) presents arguments that some "true" type 2 sources, i.e., having no obscured BLR, do exist among AGNs with $L \lesssim 10^{42} \mathrm{ergs} \mathrm{s}^{-1}$ (see also Ho et al. 1997). Both findings have a simple explanation if upon further decreases in $L$, the suppression of mass outflow spreads radially inward from the disk's dusty, molecular region into its atomic, ionized zone. Then the torus disappearance, i.e., removal of the toroidal obscuration by the dusty wind, would be followed by a diminished outflow from the inner ionized zone and disappearance of the BLR at lower, still to be determined, luminosities.

Within the framework of a hydromagnetic wind, $\dot{M}_{w} \propto \dot{M}_{\text {acc }}$, and expressing the luminosity in terms of $M_{\text {acc }}$ in equation (1), $\dot{M}_{w, \mathrm{cl}} \propto\left(\epsilon \dot{M}_{\mathrm{acc}}\right)^{1 / 2}$. The torus cloud and total outflow rates both vary in the same direction as the mass accretion rate. In contrast, Ho (2002) finds that the radio loudness of AGNs is inversely correlated with the mass accretion rate (see also Greene et al. 2006). That is, when $\dot{M}_{\text {acc }}$ is decreasing, the torus cloud and total outflow rates are also decreasing, while the radio loudness is increasing. It seems that the AGN switches its main dynamic channel for release of accreted mass from torus outflow at higher luminosities to radio jets at lower ones, with a certain degree of overlap. As also noted by Greene et al., X-ray binaries display a similar behavior, switching between radio-quiet states of high X-ray emission and radio-loud states with low X-ray emission (see Fender et al. 2004).

We have greatly benefited from discussions with many colleagues but wish to single out for special thanks remarks by Reinhard Genzel, Chris Henkel, Ari Laor, and Dani Maoz. This work was performed while M. E. spent a most enjoyable sabbatical at LAOG, Grenoble. Partial support by NASA and NSF is gratefully acknowledged.

\section{REFERENCES}

Arav, N., Shlosman, I., \& Weymann, R. J., eds. 1997, ASP Conf. Ser. 128, Mass Ejection from Active Galactic Nuclei (San Francisco: ASP) Antonucci, R. 1993, ARA\&A, 31, 473

Barth, A. J., Filippenko, A. V., \& Moran, E. C. 1999, ApJ, 525, 673

Blandford, R. D., \& Payne, D. G. 1982, MNRAS, 199, 883

Bottorff, M., Korista, K. T., \& Shlosman, I. 2000, ApJ, 537, 134

Bottorff, M., Korista, K. T., Shlosman, I., \& Blandford, R. D. 1997, ApJ, 479, 200

Chiaberge, M., Capetti, A., \& Celotti, A. 1999, A\&A, 349, 77

Davies, R. I., et al. 2006, ApJ, 646, 754

de Kool, M., \& Begelman, M. C. 1995, ApJ, 455, 448

Elitzur, M. 1996, ApJ, 457, 415

2005, in Proc. QSO Hosts: Evolution and Environment, ed. P. D.

Barthel \& D. B. Sanders (Amsterdam: Elsevier), in press (astro-ph/0512025)

Elitzur, M., Hollenbach, D. J., \& McKee, C. F. 1989, ApJ, 346, 983

Elitzur, M., Nenkova, M., \& Ivezić, Ž. 2004, in ASP Conf. Ser. 320, The

Neutral ISM in Starburst Galaxies, ed. S. Aalto, S. Hüttemeister, \& A. Pedlar (San Francisco: ASP), 242

Elvis, M. 2004, in ASP Conf. Ser. 311, AGN Physics with the Sloan Digital Sky Survey, ed. G. T. Richards \& P. B. Hall (San Francisco: ASP), 109

Emmering, R. T., Blandford, R. D., \& Shlosman, I. 1992, ApJ, 385, 460 (EBS92)

Everett, J. 2005, ApJ, 631, 689

Fender, R. P., Belloni, T. M., \& Gallo, E. 2004, MNRAS, 355, 1105

Ferreira, J. 2006, in Jets from Young Stars: Models and Constraints, ed. J. Ferreira, C. Dougados, \& E. Whelan (Berlin: Springer), in press (astro$\mathrm{ph} / 0607216)$

Galliano, E., Alloin, D., Granato, G. L., \& Villar-Martín, M. 2003, A\&A, 412, 615

Gallimore, J. F., Henkel, C., Baum, S. A., Glass, I. S., Claussen, M. J., Prieto,

M. A., \& Von Kap-herr, A. 2001, ApJ, 556, 694

Greene, J. E., Ho, L. C., \& Ulvestad, J. S. 2006, ApJ, 636, 56
Greenhill, L. J., \& Gwinn, C. R. 1997, Ap\&SS, 248, 261

Hall, P. B., et al. 2003, ApJ, 593, 189

Ho, L. C. 2002, ApJ, 564, 120

Ho, L. C., et al. 1997, ApJS, 112, 391

Jaffe, W., et al. 2004, Nature, 429, 47

Kartje, J. F., \& Königl, A. 1996, Vistas Astron., 40, 133

Kartje, J. F., Königl, A., \& Elitzur, M, 1999, ApJ, 513, 180

Kondratko, P. T., Greenhill, L. J., \& Moran, J. M. 2005, ApJ, 618, 618

Königl, A., \& Kartje, J. F. 1994, ApJ, 434, 446

Krolik, J. H., \& Begelman, M. C. 1988, ApJ, 329, 702

Laor, A. 2003, ApJ, 590, L86

Maoz, D., Nagar, N. M., Falcke, H., \& Wilson, A. S. 2005, ApJ, 625, 699

Nenkova, M., Ivezić, Ž., \& Elitzur, M. 2002, ApJ, 570, L9

Pelletier, G., \& Pudritz, R. E. 1992, ApJ, 394, 117

Perlman, E. S., Sparks, W. B., Radomski, J., Packham, C., Fisher, R. S., Piña, R., \& Biretta, J. A. 2001, ApJ, 561, L51

Poncelet, A., Perrin, G., \& Sol, H. 2006, A\&A, 450, 483

Rees, M. J. 1987, MNRAS, 228, 47P

Risaliti, G., Elvis, M., Fabbiano, G., Baldi, A., \& Zezas, A. 2005, ApJ, 623, L93

Risaliti, G., Elvis, M., \& Nicastro, F. 2002, ApJ, 571, 234

Schinnerer, E., Eckart, A., Tacconi, L. J., Genzel, R., \& Downes, D. 2000, ApJ, 533, 850

Schmitt, H. R., Antonucci, R. R. J., Ulvestad, J. S., Kinney, A. L., Clarke, C. J., \& Pringle, J. E. 2001, ApJ, 555, 663

Shlosman, I., Begelman, M. C., \& Frank, J. 1990, Nature, 345, 679

Simpson, C. 2005, MNRAS, 360, 565

Sofue, Y., Tutui, Y., Honma, M., Tomita, A., Takamiya, T., Koda, J., \& Takeda, Y. 1999, ApJ, 523, 136

Urry, C., \& Padovani, P. 1995, PASP, 107, 803

Whysong, D., \& Antonucci, R. 2004, ApJ, 602, 116 